\title{
Overlap of IgG4-related Disease and Primary Biliary Cirrhosis Complicated with Autoimmune Thrombocytopenia
}

\author{
Mika Takasumi ${ }^{1,2}$, Masayuki Miyata ${ }^{1}$, Masahito Kuroda ${ }^{1}$, Kumiko Terashima ${ }^{1}$, \\ Kazumichi $\mathrm{Abe}^{2}$, Atsushi Takahashi ${ }^{2}$, Hiroko Kobayashi ${ }^{2}$, Kazuhiro Tazaki $^{3}$, \\ Hiroshi Watanabe ${ }^{2}$ and Hiromasa Ohira ${ }^{2}$
}

\begin{abstract}
A 63-year-old woman was referred to Fukushima Red Cross Hospital with an enlargement of the left submandibular gland and subcutaneous bleeding in the chest and legs. A diffuse enlargement of the pancreas was also detected by abdominal computed tomography, and laboratory data showed severe thrombocytopenia. She was diagnosed with IgG4-related disease (IgG4-RD) complicated with autoimmune thrombocytopenia and was treated with methylprednisolone, after which the number of platelets favorably increased. Further investigation for liver dysfunction revealed underlying primary biliary cirrhosis (PBC). We herein report a rare case of IgG4-RD overlapping PBC complicated with autoimmune thrombocytopenia.
\end{abstract}

Key words: IgG4-related disease, primary biliary cirrhosis, autoimmune thrombocytopenia, prednisolone

(Intern Med 55: 1387-1392, 2016)

(DOI: 10.2169/internalmedicine.55.6202)

\section{Introduction}

IgG4-related disease (IgG4-RD) was first reported in 1993 (1), and a new disease classification was established in Japan in 2011 (2). It is a novel clinical disease entity characterized by an elevated serum IgG4 concentration and tumefaction or tissue infiltration by IgG4-positive plasma cells (3-6). A certain proportion of patients formerly diagnosed with autoimmune pancreatitis $(7,8)$ or Mikulicz's disease $(9,10)$ are believed to have had IgG4-RD. Persistent enlargement of the lacrimal and salivary glands are characteristic of Mikulicz's disease. On the other hand, primary biliary cirrhosis (PBC) is a chronic cholestatic disease with a progressive course, characterized by the presence of serum anti-mitochondrial antibodies and histological nonsuppurative destructive cholangitis. $\mathrm{PBC}$ is an autoimmune liver disease distinct from IgG4-related sclerosing cholangitis (IgG4-
$\mathrm{SC}$ ), which is classified as a biliary manifestation of IgG4RD. However, a few cases of overlapping PBC and IgG4SC have been reported (11-13). Although autoimmune thrombocytopenia is a well-defined autoimmune disease, its association with IgG4-RD or PBC is rare. We herein describe a rare case of overlapping IgG4-RD and PBC complicated with autoimmune thrombocytopenia and discuss the possible mechanisms underlying the association of these three diseases.

\section{Case Report}

A 63-year-old woman was referred to our department with an enlargement of the left submandibular gland and purpura on the chest and legs in December 2013. Her height and weight were $153 \mathrm{~cm}$ and $56 \mathrm{~kg}$, respectively. The patient's vital signs were stable: blood pressure of 135/75 $\mathrm{mmHg}$, heart rate of 73 beats per minute, body temperature

\footnotetext{
${ }^{1}$ Department of Internal Medicine and Gastroenterology, Fukushima Red Cross Hospital, Japan, ${ }^{2}$ Department of Gastroenterology and Rheumatology, Fukushima Medical University School of Medicine, Japan and ${ }^{3}$ Department of Pathology, Fukushima Medical University School of Medicine, Japan

Received for publication July 17, 2015; Accepted for publication August 16, 2015

Correspondence to Dr. Mika Takasumi, paper@fmu.ac.jp
} 
Table. Laboratory Data at Admission.

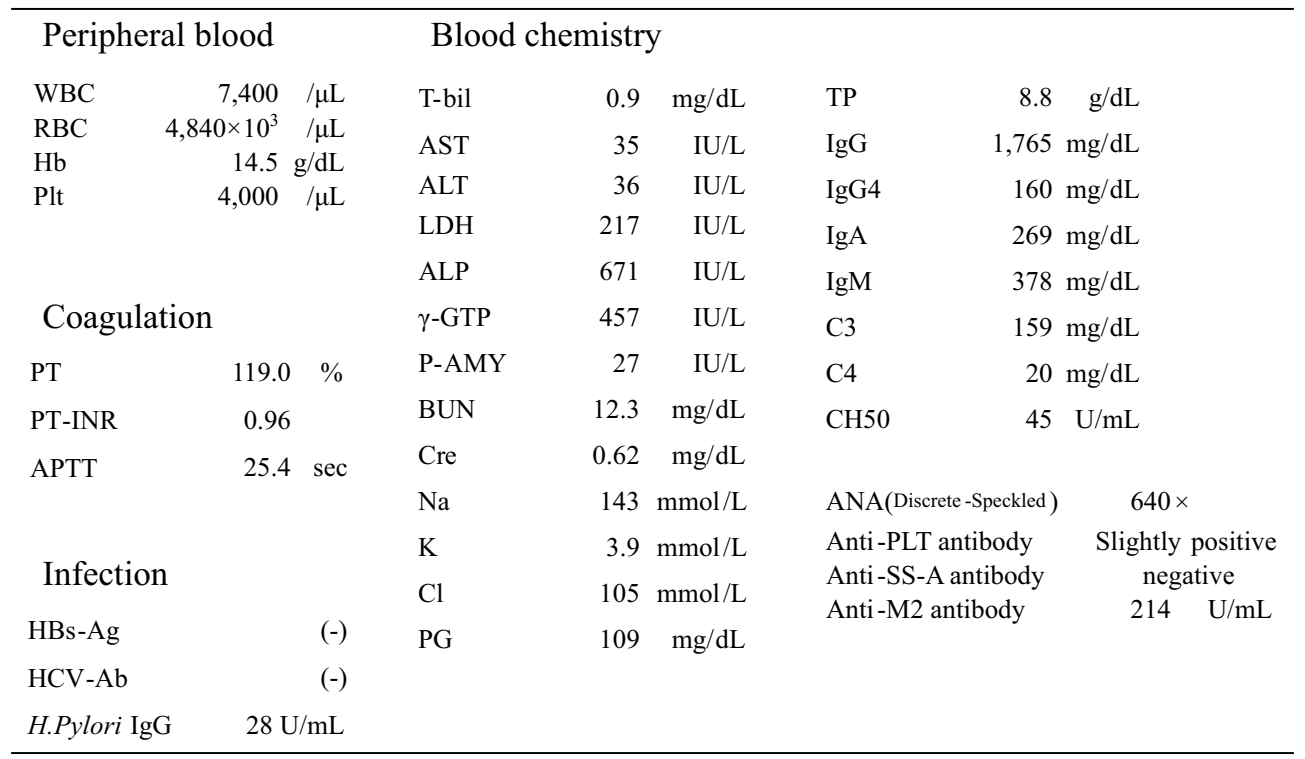

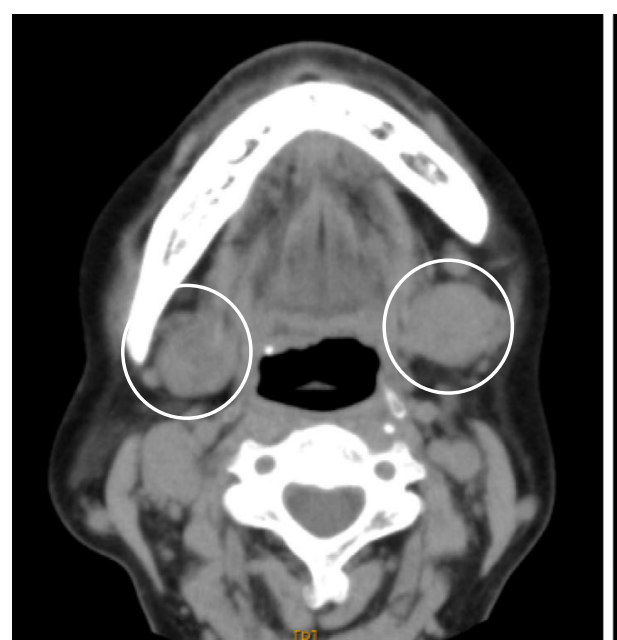

a

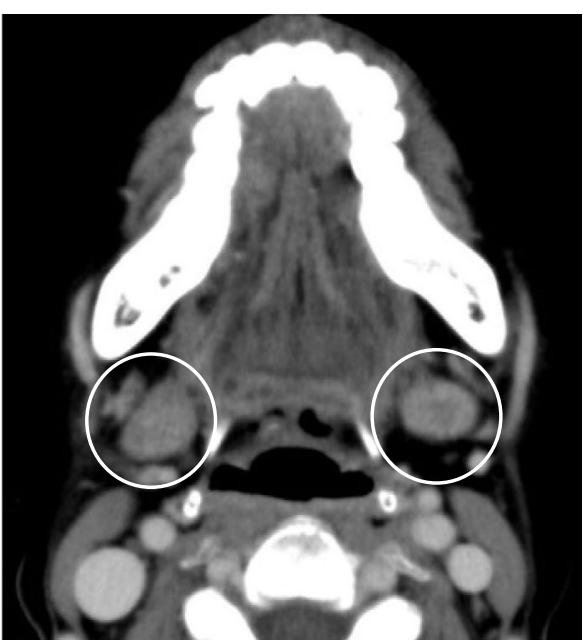

$\mathbf{b}$

Figure 1. CT of the submandibular gland. a: At admission: Non-contrast CT revealed enlargement of the bilateral submandibular gland. b: Two weeks after the beginning of PSL treatment: Contrastenhanced CT revealed decreased swelling of the bilateral submandibular gland.

of $36.2^{\circ} \mathrm{C}$, and $\mathrm{SpO}_{2}$ of $100 \%$ (room air). Laboratory data (Table) showed severe thrombocytopenia, with a platelet (PLT) count of $4,000 / \mu \mathrm{L}$. A coagulofibrinolysis test showed no abnormalities. Her anti-PLT antibody was slightly positive. The serum levels of $\gamma$-glutamyl transpeptidase $(\gamma$-GTP), alkaline phosphatase, aspartate aminotransferase (AST), and alanine aminotransferase (ALT) were high. Serum IgG was elevated at $3,160 \mathrm{mg} / \mathrm{dL}$, and $\mathrm{IgG} 4$ was elevated at $160 \mathrm{mg}$ / $\mathrm{dL}$ (normal range 4.8-105 $\mathrm{mg} / \mathrm{dL}$ ). The serum IgM level was also elevated at $378 \mathrm{mg} / \mathrm{dL}$ (normal range $46-260 \mathrm{mg} / \mathrm{dL}$ ). The complement level, which included $\mathrm{C} 3, \mathrm{C} 4$, and $\mathrm{CH} 50$, was not low. No apparent biliary dilatation or structural abnormalities of the liver were observed. However, antinuclear antibody was positive at $640 \times$ in a discrete speckled pattern and cytoplasmic antibody was positive at $80 \times$, while
anti-SS-A antibody was negative. Helicobacter pylori $(H$. pylori) $\mathrm{IgG}$ was positive at $28 \mathrm{U} / \mathrm{mL}$. Computed tomography (CT) revealed the enlargement of both submandibular glands (Fig. 1a) and diffuse enlargement of the pancreas (Fig. 2a). The enlargement of the submandibular gland was bilateral, although only the left side was palpable. No dilation of the bile and pancreatic ducts was observed.

A differential count of the bone marrow fluid obtained by aspiration was within normal limits with a slight increase of megakaryocytes, although atypical cells were not detected (Fig. 3a). Immunostaining for CD138 and IgG4 did not reveal an increase of IgG4-producing plasma cells in the bone marrow (Fig. 3b and c). From these findings, IgG4-RD and autoimmune thrombocytopenia was diagnosed, although tumefaction or tissue infiltration by IgG4-positive plasma cells 


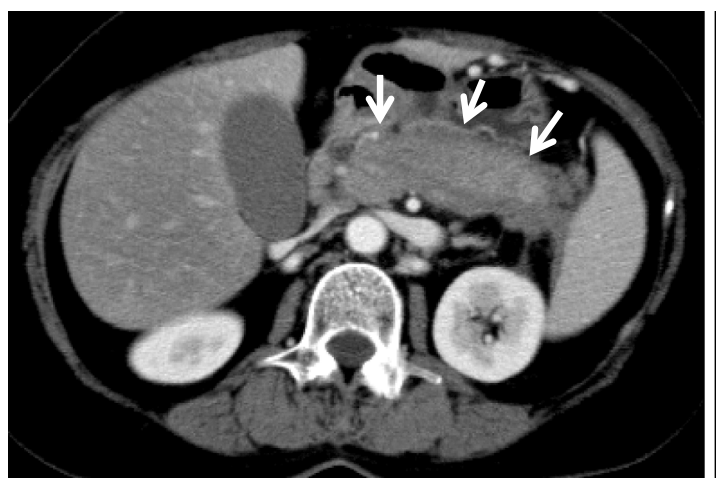

$\mathbf{a}$

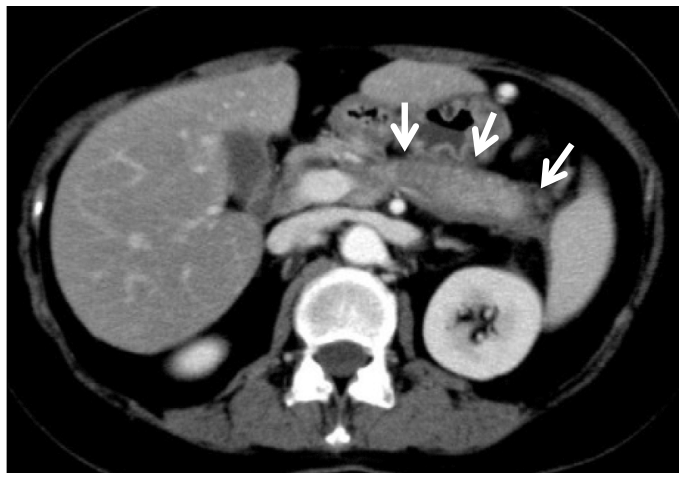

b

Figure 2. CT of the pancreas. a: At admission: Contrast-enhanced CT revealed diffuse enlargement of the pancreas as shown by arrows. Dilation of both bile and pancreatic ducts was not seen. $b$ : Two weeks after the beginning of PSL treatment: Contrast-enhanced CT revealed a decrease in diffuse swelling of the pancreas.

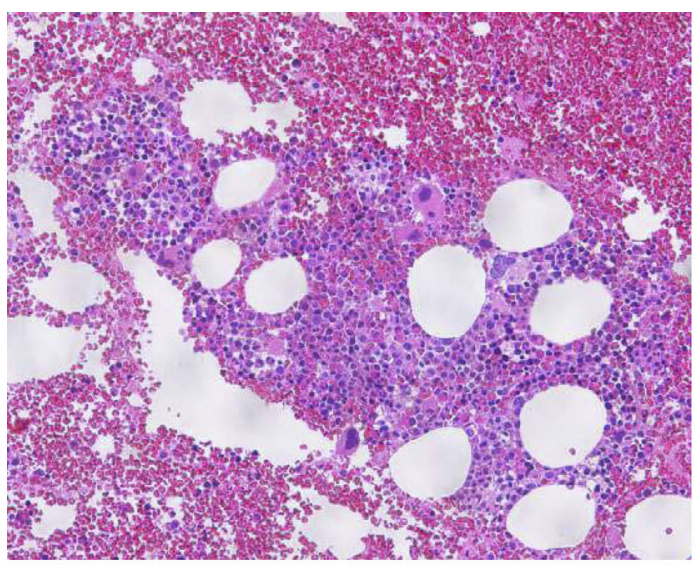

a

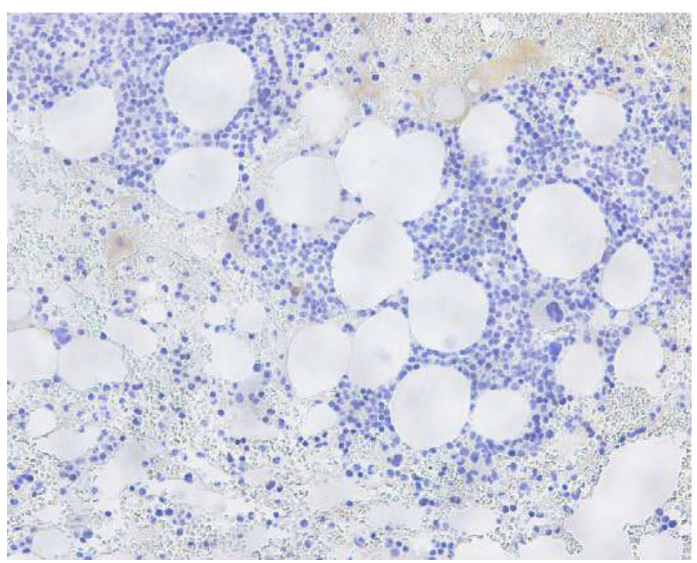

C

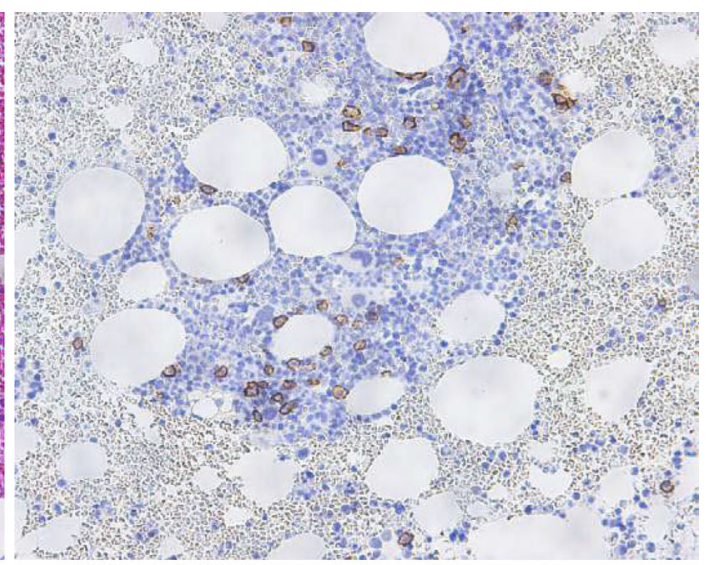

b

Figure 3. Pathological findings of the bone marrow fluid obtained by aspiration. a: Hematoxylin and Eosin staining (200x): A differential count of the bone marrow fluid was within normal limits with a slight increase in the number of megakaryocytes. Atypical cells were not detected. b: Immunostaining of CD138 (200x): The number of CD138-positive plasma cells was approximately 5\%, which remained small. c: Immunostaining of IgG4 (200x): IgG4-producing plasma cells in the bone marrow were not detected. 
a

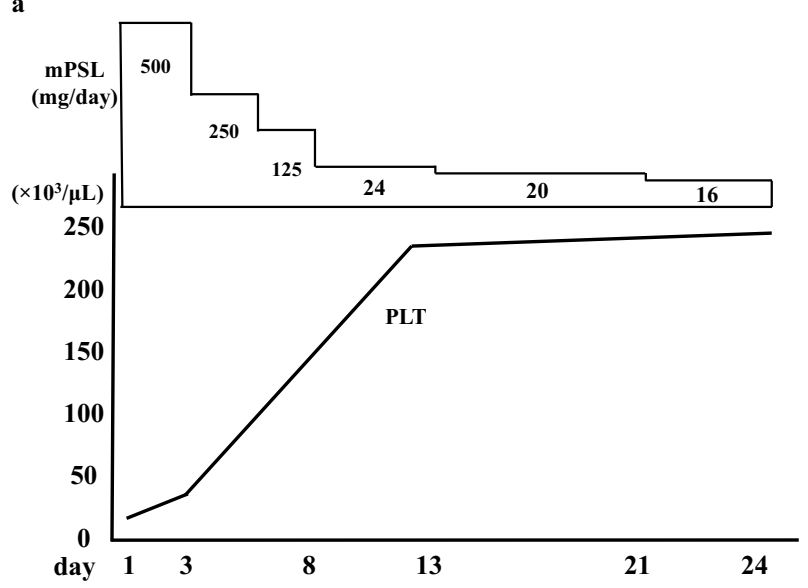

b

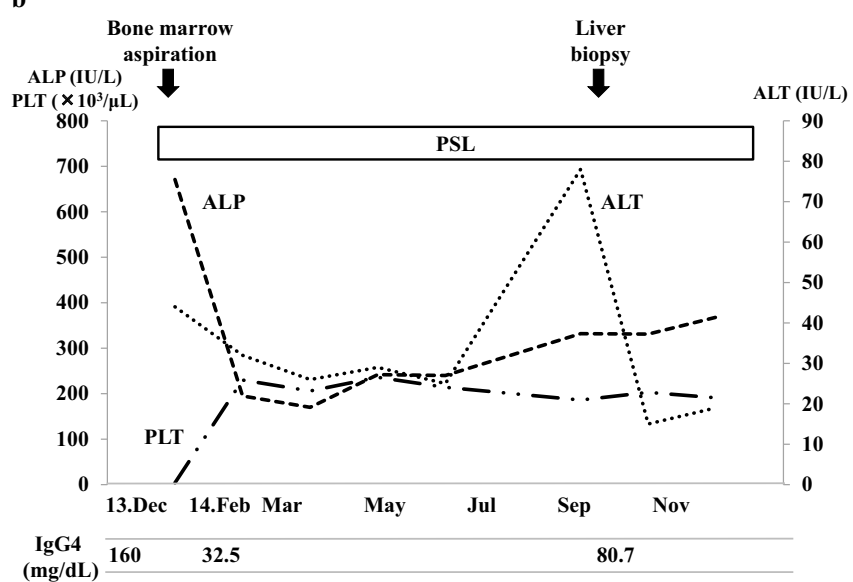

Figure 4. The clinical course of the patient.

could not be established due to severe thrombocytopenia.

Treatment with methylprednisolone (mPSL) semi-pulse (500 mg/body/day) was started for autoimmune thrombocytopenia. Because the PLT count was elevated to more than $30 \times 10^{3} / \mu \mathrm{L}$ within three days, we tapered mPSL to half the dose (Fig. 4a). Both total IgG and IgG4 were decreased to within normal limits within two months (Fig. 4b). Two weeks after beginning treatment with MPSL, CT showed a marked improvement of diffuse swelling of the bilateral submandibular gland and pancreas (Fig. 1b, 2b). The patient also received a $H$. pylori eradication 7-day course of triple therapy of lansoprazole, amoxicillin, and clarithromycin. Successful eradication was assessed by the urea breath test. Although the serum levels of AST, ALT, alkaline phosphatase (ALP), and $\gamma$-GTP decreased temporarily, $\gamma$-GTP did not decrease to within normal levels, and AST and ALT reelevated six months after beginning mPSL treatment (Fig. 4b). The serum level of anti-mitochondrial M2 (anti-M 2) antibody was measured in order to determine a cause related to liver dysfunction, and it was elevated at $214 \mathrm{U} / \mathrm{mL}$ (normal range, $<7 \mathrm{U} / \mathrm{mL}$ ). Serologic markers for hepatitis $B$ and $\mathrm{C}$ viruses were negative. Magnetic resonance cholangiopancreatography revealed neither strictures of the lower common bile duct nor a segmental stricture. Because underlying $\mathrm{PBC}$ was suspected at this time, a liver biopsy was performed. The liver specimen showed infiltration of lymphocytes in the portal area but did not reveal any obvious bile duct destruction by lymphocytes. IgG4-positive plasma cells were not observed in this specimen. Early-phase chronic nonsuppurative destructive cholangitis (CNSDC) was suggested (Fig. 5). Liver dysfunction was improved by the administration of ursodeoxycholic acid after the liver biopsy.

\section{Discussion}

IgG4-RD and autoimmune thrombocytopenia (ITP) were initially diagnosed in this case. However, PBC was subsequently suspected after the administration of mPSL therapy. Although a liver biopsy did not reveal any obvious bile duct destruction due to CNSDC, PBC could be diagnosed by an elevated serum anti-M2 antibody level, which has a high sensitivity and specificity for the diagnosis of PBC $(14,15)$. Elevation of serum IgM also suggested the existence of PBC. Magnetic resonance cholangiopancreatography was performed, but the presence of IgG4-SC was not determined. IgG4-SC generally reveals segmental strictures, long strictures with prestenotic dilatation, and strictures of the lower common bile duct. Biliary strictures in IgG4-SC typically respond to steroids, and complete resolution of the strictures and/or normalization of liver tests has been observed in approximately two-thirds of patients, whereas improvement was seen in the remaining one-third (16). It was difficult to rule out the presence of $\mathrm{IgG} 4-\mathrm{SC}$ in our case treated by mPSL.

An underlying common pathogenesis may exist in these three diseases. First, regarding the common pathogenesis of PBC and IgG4-RD, Shimoda et al. (17) reported the role of Toll-like receptors and natural killer (NK) cells in PBC patients. Toll-like receptor 4 ligand (TLR4-L)-stimulated NK cells destroy autologous biliary epithelial cells in the presence of interferon (IFN)- $\alpha$ synthesized by TLR 3 ligand (TLR3-L)-stimulated monocytes. According to Shimoda et al., IFN- $\alpha$ production by hepatic monocytes was significantly increased in patients with PBC. The cytotoxic activity of hepatic NK cells was also increased compared to controls. However, this only occurred when the NK cells were prepared following ligation of both TLR3-L- and TLR4-Lstimulated liver mononuclear cells. On the other hand, Watanabe et al. (18) reported that activation of TLR and nucleotide-binding oligomerization domain-like receptors enhanced IgG4 responses in autoimmune pancreatitis. Stimulation with TLR3 and TLR4 ligands enhances IgG4 production by peripheral blood mononuclear cells in IgG4-RD patients. These consequences suggest a common pathogenesis in IgG4-RD and PBC through abnormal innate immune responses against microbial antigens.

Next, we examined the pathogenesis of PBC and autoimmune thrombocytopenia. Panzer et al. (19) reported that autoantibodies eluted from a patient with PBC and autoimmune thrombocytopenia, precipitate glycoprotein IIb/IIIa of 


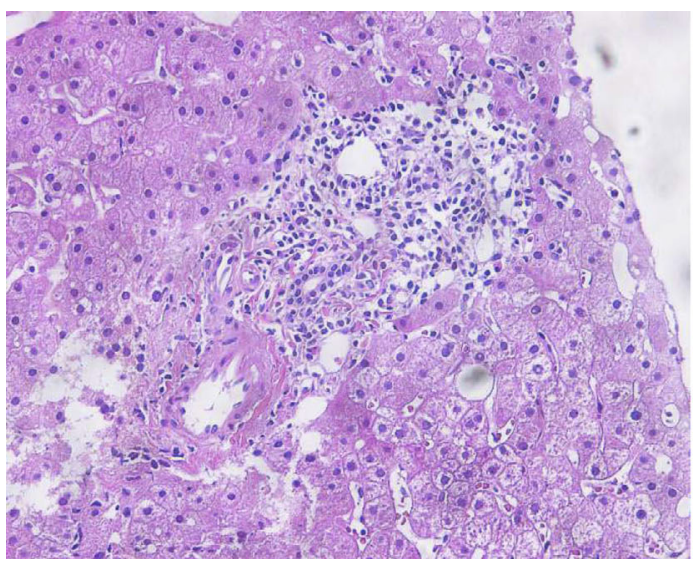

a

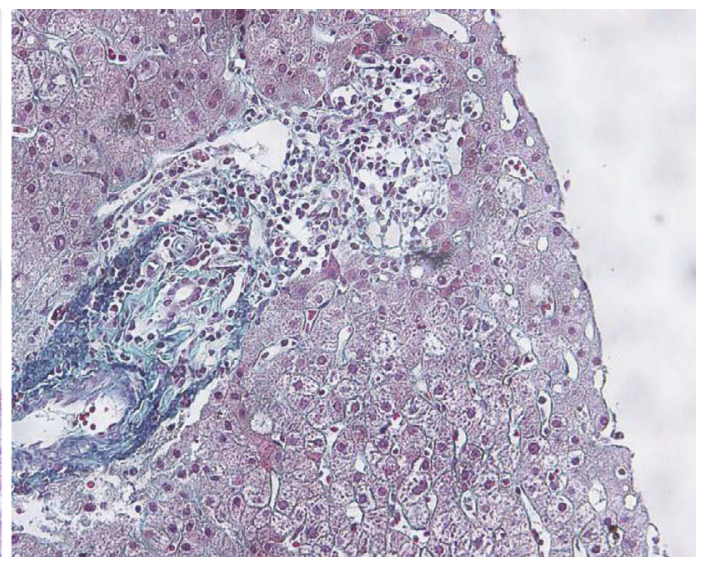

b

Figure 5. Pathological findings of the liver obtained by a needle biopsy. a: Hematoxylin and Eosin staining (200x). b: Elastica-Masson staining (200x). A liver specimen showed infiltration of lymphocytes in the portal area, but no obvious bile duct destruction by lymphocytes was found. There was slight deformation of the bile duct. Taken together, early phase chronic nonsuppurative destructive cholangitis (CNSDC) could not be ruled out.

autologous and allogeneic PLT and bind to an epitope of the rat $70-\mathrm{kDa}$ mitochondrial protein M2. Furthermore, an in silico analysis of published peptide sequences of the mitochondrial protein and glycoprotein IIb/IIIa showed partial amino acid sequence homology, suggesting the possibility of a common antibody-binding site. From these findings, PBCrelated autoantibodies might cross-react with PLT surface autoantigens in ITP (20).

A common underlying pathogenesis of IgG4-RD and autoimmune thrombocytopenia was considered. However, the role of the IgG4 antibody response in the immunopathogenesis of IgG4-RD is poorly understood. Moreover, serum IgG4 levels fluctuate along with the state of IgG4-RD. A few cases have been reported regarding the association between IgG4-RD, especially with autoimmune pancreatitis, and autoimmune thrombocytopenia (21-24). In the present case, no significant increase in the number of IgG4-positive cells or decrease in megakaryocytes in the bone marrow was observed. This suggests that the cause of thrombocytopenia was not a decrease in megakaryocytes in relation to the proliferation of IgG4-positive cells in the bone marrow, but the presence of anti-PLT antibody. Patients with ITP have antiPLT autoantibodies that are most frequently directed against PLT glycoproteins IIb/IIIa or Ib/IX/V. Chan et al. (25) reported that the most common subclass of anti-IIb/IIIa antibodies in ITP patients was IgG1. Murase et al. (22) reported an IgG subclass of a PLT antibody to be potentially IgG4. While this suggested a role of IgG4 as an anti-PLT antibody in IgG4-RD patients, this finding is controversial because IgG4-RD is rarely complicated with thrombocytopenia.

H. pylori infection could also contribute to IgG4-RD and ITP. The peptide showed homology with an amino acid sequence between $H$. pylori $\alpha$-carbonic anhydrase $(\alpha-\mathrm{CA})$ and human CA type II and between $H$. pylori plasminogenbinding protein and human ubiquitin-protein ligase E3 com- ponent n-recognin 2, an enzyme highly expressed in acinar cells of the pancreas $(26,27)$. It has been suggested that this molecular mimicry can lead to immunological crossreactivity. Furthermore, CagA antigen of $H$. pylori may induce anti-GPIIb/IIIa antibody production by a molecular mimicry mechanism (28), suggested a contribution to ITP. Although there is molecular mimicry between the urease beta antigen of $H$. pylori and E2 subunit of the pyruvate dehydrogenase complex of the major mitochondrial autoepitope, this homology does not lead to immunological crossreactivity $(29,30)$.

In summary, it could be inferred that serum anti-M2 antibody from PBC or IgG4 from IgG4-RD recognized PLT as an antigen, subsequently inducing severe thrombocytopenia in our patient. In addition, IgG4 production may be enhanced by PBC. H. pylori infection could also contribute to IgG4-RD and ITP. We herein experienced a rare case of overlapping IgG4-RD and PBC complicated with autoimmune thrombocytopenia. A combination of these three diseases suggests that common immunogenetic factors might be involved in the development of IgG4-RD, PBC, and autoimmune thrombocytopenia.

The authors state that they have no Conflict of Interest (COI).

\section{References}

1. Suzuki S, Kida S, Ohira Y, et al. A case of Sjögren's syndrome accompanied by lymphadenopathy and IgG4 hypergammaglobulinemia. Ryumachi 33: 249-254, 1993.

2. Umehara H, Okazaki K, Masaki Y, et al. Comprehensive diagnostic criteria for IgG4-related disease (IgG4-RD), 2011. Mod Rheumatol 22: 21-30, 2012.

3. Okazaki K, Umehara $\mathrm{H}$. Are classification criteria for IgG4-RD now possible? The concept of IgG4-related disease and proposal of comprehensive diagnostic criteria in Japan. Int J Rheumatol 
2012: 357071, 2012.

4. Umehara H. A new clinical entity: IgG4-related disease (IgG4RD) discovered in the 21st century. Intern Med 51: 821-822, 2012.

5. Umehara H, Okazaki K, Masaki Y, et al. A novel clinical entity, IgG4-related disease (IgG4RD): general concept and details. Mod Rheumatol 22: 1-14, 2012.

6. Umehara H, Sato T, Nakamura T, Tanaka M. IgG4-related disease -a new clinical entity established by all Japan IgG4 team in 21st century. Arerugi 62: 1591-1597, 2013.

7. Hamano H, Kawa S, Horiuchi A, et al. High serum IgG4 concentrations in patients with sclerosing pancreatitis. $\mathrm{N}$ Engl $\mathrm{J}$ Med 344: 732-738, 2001.

8. Yoshida K, Toki F, Takeuchi T, Watanabe S, Shiratori K, Hayashi N. Chronic pancreatitis caused by an autoimmune abnormality. Proposal of the concept of autoimmune pancreatitis. Dig Dis Sci 40: 1561-1568, 1995.

9. Yamamoto M, Ohara M, Suzuki C, et al. Elevated IgG4 concentrations in serum of patients with Mikulicz's disease. Scand J Rheumatol 33: 432-433, 2004

10. Yamamoto M, Takahashi H, Naishiro Y, et al. Mikulicz's disease and systemic IgG4-related plasmacytic syndrome (SIPS). Nihon Rinsho Meneki Gakkai Zasshi (Jpn J Clin Immunol) 31: 1-8, 2008 (in Japanese, Abstract in English).

11. Naitoh I, Nakazawa $T$, Hayashi K, et al. A case of IgG4-related sclerosing cholangitis overlapped with primary biliary cirrhosis. Intern Med 51: 1695-1699, 2012.

12. Takemoto R, Miyake $Y$, Harada K, et al. Overlap of IgG4-related sclerosing cholangitis and primary biliary cirrhosis. Intern Med 53: $1429-1433,2014$

13. Wiegand J, Neid M, Kaiser T, et al. Coexistence of autoimmune pancreatitis and primary biliary cirrhosis in a Caucasian patient - a rare cause of cholestasis. Z Gastroenterol 44: 1227-1229, 2006.

14. Bassendine MF, Collins JD, Stephenson J, Saunders P, James OF. Platelet associated immunoglobulins in primary biliary cirrhosis: a cause of thrombocytopenia? Gut 26: 1074-1079, 1985.

15. Berg PA, Klein R, Lindenborn-Fotinos J, Kloppel W. ATPaseassociated antigen (M2): marker antigen for serological diagnosis of primary biliary cirrhosis. Lancet 2: 1423-1426, 1982.

16. Ghazale A, Chari ST, Zhang L, et al. Immunoglobulin G4associated cholangitis: clinical profile and response to therapy. Gastroenterology 134: 706-715, 2008.

17. Shimoda S, Harada K, Niiro H, et al. Interaction between Toll-like receptors and natural killer cells in the destruction of bile ducts in primary biliary cirrhosis. Hepatology 53: 1270-1281, 2011.

18. Watanabe $T$, Yamashita $K$, Fujikawa $S$, et al. Involvement of activation of toll-like receptors and nucleotide-binding oligomerization domain-like receptors in enhanced IgG4 responses in autoim- mune pancreatitis. Arthritis Rheum 64: 914-924, 2012.

19. Panzer S, Penner E, Nelson PJ, Prochazka E, Benda H, Saurugger PN. Identification of the platelet glycoprotein IIb/IIIa complex as a target antigen in primary biliary cirrhosis-associated autoimmune thrombocytopenia. Evidence that platelet-reactive autoantibodies can also bind to the mitochondrial antigen M2. J Autoimmun 3: 473-483, 1990.

20. Toshikuni N, Yamato R, Kobashi H, et al. Association of primary biliary cirrhosis with idiopathic thrombocytopenic purpura. World J Gastroenterol 14: 2451-2453, 2008.

21. Fukushima H, Katou E, Nagayama K, Shirachi A, Sata M. A case of autoimmune pancreatitis complicated with immune thrombocytopenia. Jpn J Gastroenterol 103: 661-666, 2006 (in Japanese, Abstract in English).

22. Murase K, Matsunaga T, Hayashi T, et al. Successful treatment of autoimmune pancreatitis complicated with autoimmune thrombocytopenia and interstitial pneumonia by prednisolone. Intern Med 47: 1033-1038, 2008.

23. Nakamura A, Funatomi H, Katagiri A, et al. A case of autoimmune pancreatitis complicated with immune thrombocytopenia during maintenance therapy with prednisolone. Dig Dis Sci 48: 1968-1971, 2003.

24. Seko S, Taniguchi T, Nishikawa H, et al. A case of autoimmune pancreatitis associated with immune thrombocytopenia. Nihon Shokaki Naishikyo Gakkai Zasshi (Gastroenterol Endosc) 42: 192197, 2000 (in Japanese, Abstract in English).

25. Chan H, Moore JC, Finch CN, Warkentin TE, Kelton JG. The IgG subclasses of platelet-associated autoantibodies directed against platelet glycoproteins IIb/IIIa in patients with idiopathic thrombocytopenic purpura. Br J Haematol 122: 818-824, 2003.

26. Frulloni L, Lunardi C, Simone R, et al. Identification of a novel antibody associated with autoimmune pancreatitis. N Engl J Med 361: 2135-2142, 2009.

27. Bulajic M, Panic N, Lohr JM. Helicobacter pylori and pancreatic diseases. World J Gastrointest Pathophysiol 5: 380-383, 2014.

28. Cheng YS, Kuang LP, Zhuang CL, Jiang JD, Shi M. Effects of cytotoxin-associated gene A (CagA) positive Helicobacter pylori infection on anti-platelet glycoprotein antibody producing B cells in patients with primary idiopathic thrombocytopenic purpura (ITP). Pak J Med Sci 31: 121-126, 2015.

29. Bogdanos DP, Baum H, Gunsar F, et al. Extensive homology between the major immunodominant mitochondrial antigen in primary biliary cirrhosis and Helicobacter pylori does not lead to immunological cross-reactivity. Scand J Gastroenterol 39: 981-987, 2004.

30. Durazzo M, Rosina F, Premoli A, et al. Lack of association between seroprevalence of Helicobacter pylori infection and primary biliary cirrhosis. World J Gastroenterol 10: 3179-3181, 2004.
(C) 2016 The Japanese Society of Internal Medicine http://www.naika.or.jp/imonline/index.html 